measures. At each time-point MEDD, DEDD and ACL were calculated. Multilevel modelling was used to investigate independent associations between MEDD, DEDD and ACL, and cognitive and gastrointestinal symptoms, quality of life, performance status and survival.

Results Cognitive and gastrointestinal symptoms, performance status, and quality of life worsened over time. In the adjusted multilevel analysis significance remained for worsening performance status (MEDD, $\mathrm{p}=0.001$; DEDD, $\mathrm{p}<0.001$; ACL $\mathrm{p}=0.035$ ) and shorter time to death (MEDD, $\mathrm{p}<0.001$; ACL, $\mathrm{p}<0.01$ ).

Conclusion Commonly used palliative medications were associated with deteriorating performance status and shorter time to death. This analysis highlights the importance of adjusting for other variables, including other medication when exploring medication-related harms. An understanding of the risk-benefit balance of medications is needed to maximise net benefit for patients. Future work to delineate interactions between classes of drugs and drug-related harms and to evaluate early assessment and management of side-effects is needed in order to maximise net benefit.

\section{0-4 USE OF ACTIGRAPHY FOR PROGNOSTICATION IN CANCER PATIENTS}

Andrew Davies.

$10.1136 /$ bmispcare-2017-00133.4

\section{0-5 A SYSTEMATICALLY STRUCTURED REVIEW ON BIOMARKERS OF DYING IN CANCER PATIENTS AT THE END OF LIFE; AN EXPLORATION OF POTENTIAL MECHANISMS FOR THE BIOLOGY OF DYING}

${ }^{1}$ Victoria Reid, ${ }^{2}$ Rachael McDonald, ${ }^{1}$ Amara Callistus Nwosu, ${ }^{1}$ Stephen R Mason, ${ }^{3}$ Chris Probert, ${ }^{1}$ John E Ellershaw, ${ }^{1}$ Seamus Coyle. ${ }^{1}$ The Marie Curie Palliative Care Institute, University of Liverpool, Liverpool, UK; ${ }^{2}$ Renal Medicine, Aintree University Hospital NHS Foundation Trust, Liverpool, UK; ${ }^{3}$ Department of Gastroenterology, University of Liverpool, UK

\subsection{6/bmjspcare-2017-00133.5}

Background The Neuberger review made a number recommendation to improve end of life care, including research into the biology of dying. An important aspect of the biology of dying is the identification of biomarkers of the dying process. Biomarkers have the potential to assist clinicians in recognising dying, in particular how to distinguish dying from reversible acute deterioration.

Objectives To critically appraise the existing literature on prognostic biological factors that impact survival in advanced cancer patients in the last days, weeks or months of life; to identify prognostic models for advanced cancer patients, which could assist clinicians to prognosticate in the last days, weeks or months of life; and to identify candidate biomarkers of the dying process that can be measured serially in bodily fluids.

Methods A systematically structured review was conducted using three electronic databases. A hand search of six peerreviewed journals and conference abstracts was also conducted. Studies reporting biomarkers of dying in cancer patients with a median survival of $\leq 90$ days, and post-mortem studies were included.
Results 30 articles were included. There is grade A evidence for the following biological factors: serum CRP, WBC count, lymphopaenia, serum sodium, urea, ALP and hypoalbuminaemia. An additional nine prognostic factors were identified with grade B evidence including: thrombocytopaenia, elevated vitamin B12, hyperbilirubinaemia, hypocholesterolaemia, elevated AST, ALT, LDH and INR. In the last two weeks of life, a number of biomarkers have been identified but limitations exist. No post-mortem studies met the inclusion criteria.

Conclusion The biology of dying is an important area for future research interest. The evidence base to date is largely focused on symptoms, signs and prognostic factors. We identify a number common themes shared amongst advanced cancer patients, candidate biomarkers of dying, and areas for future research including non-invasive research methodologies.

\section{0-6 A CLUSTER RANDOMISED TRIAL OF CLINICALLY ASSISTED HYDRATION AT THE END OF LIFE}

${ }^{1,2}$ Andrew Davies, 'Melanie Waghorn, ${ }^{2}$ Sigurd Johnsen. 'Royal Surrey County Hospital, Guildford, UK; ${ }^{2}$ University of Surrey, Guildford, UK

\subsection{6/bmjspcare-2017-00133.6}

Background Clinically-assisted hydration (CAH) at the end-oflife is one of the most contentious issues in medicine, partly due to the fact that there is no good data to support/refute its use in this scenario.

Methods The study was a cluster randomised trial (feasibility study) comparing $\mathrm{CAH}$ with oral care in patients with advanced cancer receiving end-of-life care under palliative care teams in 12 hospices/hospitals in the UK. The main outcomes related to the feasibility of conducting a definitive study, whilst the clinical outcomes included the prevalence of end-oflife care symptoms (particularly hyperactive delirium), adverse effects, and overall survival.

Results 200 patients were recruited in 1 year, and all feasibility criteria were achieved. The prevalence of delirium was similar in the two groups, although the onset of delirium was delayed in the CAH group (112 hour versus 58 hour). Similar results were seen for excess respiratory secretions ("death rattle"). Median survival was greater in the $\mathrm{CAH}$ group (i.e. 5 days versus 3 days). Thirty-eight percent patients discontinued $\mathrm{CAH}$ due to perceived adverse effects (e.g. localised swelling, respiratory secretions).

Conclusion Interventional trials are possible in patients at the end-of-life, but the methodology needs to be somewhat adapted. The results of the feasibility study suggest that $\mathrm{CAH}$ may have a positive influence of end-of-life problems, and possibly survival. However, a larger/definitive study is required to confirm these findings. CAH is associated with adverse effects in some patients, but these may be less than perceived by palliative care specialists.

\section{0-7 ROBOTIC TECHNOLOGY AND PALLIATIVE CARE EDUCATION: THE DEVELOPMENT OF A 'NAO ROBOT' COMPUTER PROGRAM}

${ }^{1}$ Bethany Sturgeon, ${ }^{2}$ Terry Payne, ${ }^{3}$ Stephen Mason, ${ }^{3}$ Amara Nwosu. ${ }^{1}$ University of Bristol, Bristol, UK; ${ }^{2}$ Department of Computer Science, University of Liverpool, Liverpool, UK; ${ }^{3}$ Marie Curie Palliative Care Institute Liverpool, University of Liverpool, Liverpool, UK

10.1136/bmjspcare-2017-00133.7 
Background Robots are increasingly being used to support management in certain areas of healthcare education. However, the potential application of robotics in palliative care education or simulation has not been explored.

Aim This collaborative project between Computer Science and Palliative Care aimed to program a robot to convey emotion in response to human interaction, in order to develop a robotics program for potential use in palliative care education. Methods The Nao robot is an autonomous, programmable humanoid robot that is controlled by a Linux-based operating system. The robot has capabilities for voice recognition and sound localisation (in-built microphones), multilingual text-tospeech synthesis (in-built speakers) and vision, which include facial and shape recognition (in-built high definition cameras). The robot was programmed by a computer scientist to convey ten emotions (relaxed, anger, withdrawn/sad, lightly crying, heavy sobbing, happy/excited, scared, tired, laughing and dancing) through its posture, movement and speech, in response to human-voiced questions and interaction.

Results The robot was successfully programmed to convey the ten target emotions in response to direct questions posed by a human subject. Discussions around the robot's displayed emotions were explored (e.g. "why are you sad?") to assess the potential of human-computer interaction. The robot continues to acquire a growing lexicon of vocabulary, in addition to an increasing number actions and responses. The robot acts both autonomously and through direct instruction of the operator. Conclusions We have successfully programmed a robot to interact with humans and display emotional responses. This technology could potentially be used to develop innovative ways to engage individuals in discussion about palliative care issues and create opportunities to use robots for interactive educational activity. Consequently, further research can explore the potential to use robotic technology in palliative care for education, and to promote discussion with the public (e.g. children) and healthcare professionals.

\section{0-8 IN SILICO MODELLING OF THE PLASMA MORPHINE CONCENTRATION AND THE LEGAL DRIVING LIMIT}

${ }^{1}$ Jason W Boland, ${ }^{1}$ Miriam J Johnson, ${ }^{2}$ David J Berry. ${ }^{1}$ Hull York Medical School, University of Hull, Hull, UK; ${ }^{2}$ School of Medicine, Pharmacy and Health, Durham University, Durham, UK

\subsection{6/bmjspcare-2017-00133.8}

Background The legal blood morphine concentration for driving in England and Wales has been set at $80 \mu \mathrm{g} / \mathrm{L}$, based on consensus. There is little information regarding the doses likely to cause levels above this limit. Knowledge of the doseconcentration relationship would aid doctors' prescribing decisions and individualised advice. The aim of this study was to investigate the in silico relationship of oral morphine dose and plasma concentration in the context of a morphine plasma concentration of $80 \mu \mathrm{g} / \mathrm{L}$ in different patient groups.

Methods A dose-concentration in silico model for different genders, ages and morphine formulations was generated using Simcyp, a population-based pharmacokinetic simulator. This software simulates absorption and metabolism in a physiology based modelling platform, calculating a range of steady state dose-plasma concentrations across a diverse population. The morphine model created was validated against clinical pharmacokinetic data for oral immediate-release and modifiedrelease preparations. This model calculates only morphine concentrations not its active metabolites; in line with the driving law.

Results Older age, female gender, modified-release formulation and renal dysfunction were associated with higher plasma concentrations at steady state. Except in females over 80 years old or in people with impaired renal function, morphine doses below $120 \mathrm{mg} / \mathrm{d}$ were unlikely to result in a morphine plasma concentration above $80 \mu \mathrm{g} / \mathrm{L}$. In males less than 40 years old with normal renal function, doses up to $250 \mathrm{mg} / \mathrm{d}$ were unlikely to result in a morphine plasma concentration above $80 \mu \mathrm{g} / \mathrm{L}$. An immediate-release morphine dose taken alongside modified-release morphine leads to a higher plasma concentration.

Conclusion These derived morphine dose-concentrations could provide a reference frame for the prescribing clinician. However, the decision and communication to the patient must primarily take into account clinical judgment, the individual patient's level of impairment and insight for any given dose and plasma concentration.

\section{0-9 PHASE 3 STUDY TO EVALUATE THE EFFICACY AND SAFETY OF NALDEMEDINE FOR THE TREATMENT OF OPIOID-INDUCED CONSTIPATION (OIC) IN CANCER PATIENTS}

${ }^{1}$ Takaaki Yokota, ${ }^{2}$ Nobuyuki Katakami, ${ }^{3}$ Toshiyuki Harada, ${ }^{1}$ Yukio Tada, ${ }^{4}$ Masaru Narabayashi, ${ }^{5}$ Narikazu Boku. ${ }^{1}$ Shionogi and Co., Ltd., Osaka, Japan; ${ }^{2}$ Institute of Biomedical Research and Innovation, Kobe, Japan; ${ }^{3} \mathrm{~J}$ CHO Hokkaido Hospital, Sapporo, Japan; ${ }^{4}$ Cancer Institute Hospital of JFCR, Tokyo, Japan; ${ }^{5}$ National Cancer Centre Hospital, Tokyo, Japan

\subsection{6/bmjspcare-2017-00133.9}

Background While opioid analgesics play a central role in managing cancer pain, opioid-induced constipation (OIC) is one of the most common side effects. Naldemedine is a peripherally-acting $\mu$-opioid receptor antagonist being developed to treat OIC.

Methods Studies consisted of a 2 week randomised doubleblind placebo-controlled treatment period (DBT) followed by a 12 week open-label extension (EXT). In DBT, cancer patients with OIC, defined as $\leq 5$ spontaneous bowel movements (SBMs) 14 day before randomization, were randomised 1:1 to oral naldemedine $0.2 \mathrm{mg}$ QD or placebo. Patients who completed DBT could receive naldemedine in EXT. The primary endpoint of DBT was SBM responder rate (percentage of patients with $\geq 3 \mathrm{SBMs} /$ week and an increase from baseline of $\geq 1 \mathrm{SBM} /$ week) in the naldemedine group compared with placebo. The primary objective of EXT was to assess longterm safety.

Results A total of 193 patients were randomised in DBT, and 131 patients were enrolled in EXT. In DBT, significantly higher SBM responder rate was observed in naldemedine compared with placebo $(71.1 \%$ vs $34.4 \%$, respectively; $\mathrm{p}<0.0001)$. Naldemedine improved change from baseline in the frequency of SBMs (5.16 vs 1.54, p<0.0001), SBMs with a feeling of complete evacuation $(2.76$ vs $0.71, \mathrm{p}<0.0001)$ and SBMs without straining (3.85 vs $1.17, \mathrm{p}=0.0005)$ per week. Incidences of adverse event (AE) reported during treatment period in DBT were $44.3 \%$ and $26.0 \%$ in naldemedine and placebo, 